\title{
Acral peeling skin syndrome: A misdiagnosed entity
}

\section{Sanae Sialiti, Khallaayoune Mehdi, Mariame Meziane, Nadia Ismaili, Laila Benzekri, Karima Senouci}

Department of Dermatology, University Hospital Ibn Sina Rabat, Morocco

Corresponding author: Sanae Sialiti, MD, E-mail: dr.sialiti@gmail.com

Sir,

A three-year-old female, from a non-consanguineous marriage, with no particular antecedent, presented with non-pruritic desquamation of the lateral edges of the hands and feet evolving for the last several months, without associated functional signs. No family member was reported to suffer from the same physical condition.

Her parents reported worsening of the symptoms during the summer and when immersed in water and their improvement during the winter. A dermatological examination revealed peeling skin limited to the acral parts of the distal limbs and the lateral edges of the hands and feet (Fig. 1).

There were no signs of other skin lesions, such as oozing, suppuration, or scarring (Fig. 2), and the rest of the somatic examination was unremarkable.

Mycological sampling for dermatophytes was negative and the histopathological examination of a deep-skin biopsy revealed superficial detachment of the stratum corneum from the stratum granulosum and mild orthokeratotic hyperkeratosis.

The identification of mutations in the TGM5 gene localized in chromosome $15 \mathrm{ql} 5$ coding transglutaminase 5 could not be conducted due to lack of means and resources.

To date, the patient has evolved well with the daily application of emollient cream with a four-year followup period.

Acral peeling syndrome is an autosomal recessive genodermatosis induced by lack of cohesion in the cells

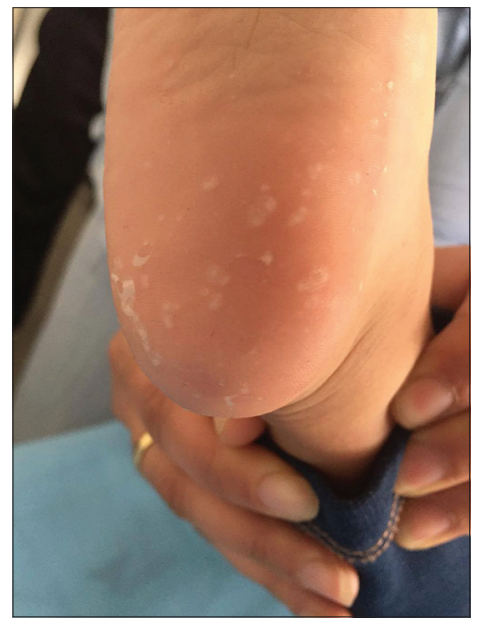

Figure 1: Flap-like desquamation of the outer lateral edge of the foot.

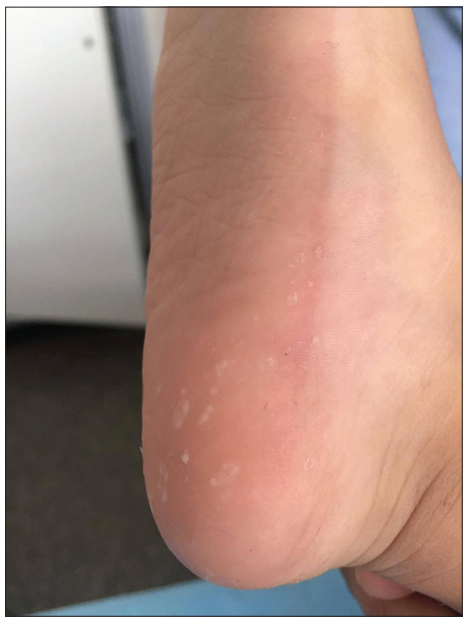

Figure 2: Slightly hyperpigmented streaks associated with peeling skin on the foot.

of the stratum corneum. Initially described in 1921 by Fox as keratolysis exfoliativa congenita [1], its exact prevalence is unknown, with a dozen cases reported in the literature.

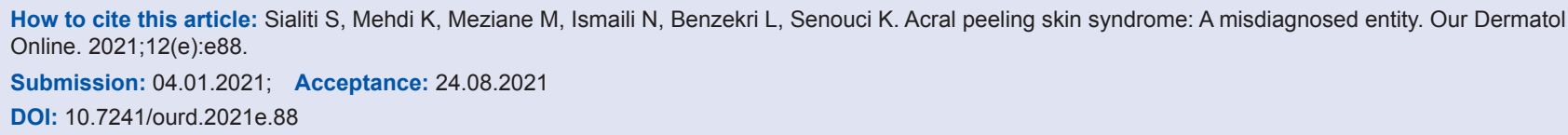


This genodermatosis begins in childhood and is clinically characterized by continuous desquamation, initially located on the extremities with a possibility of secondary generalization to the rest of the body. Two types of this entity are distinguished: inflammatory and non-inflammatory [2]. Our patient presented with the second, which may involve no erythema and peeling skin only.

Lesions disappear in winter and flare-ups appear most often in summer, especially with sweating and rubbing.

Histopathology reveals psoriasiform acanthosis, orthokeratotic hyperkeratosis, or superficial detachment between the stratum corneum and the granular layer. The dermis contains no inflammatory infiltrate [3].

Molecular studies reveal a homozygous mutation of the gene on chromosome $15 \mathrm{q} 15$ coding transglutaminase 5 , but this is not essential for diagnostic confirmation [4].

The treatment is not well codified and, being mostly symptomatic, is based on the observation of aggravating factors in association with emollients or keratolytics. UV light, methotrexate, and oral retinoids seem to be ineffective.

\section{Consent}

The examination of the patient was conducted according to the principles of the Declaration of Helsinki.

The authors certify that they have obtained all appropriate patient consent forms, in which the patients gave their consent for images and other clinical information to be included in the journal. The patients understand that their names and initials will not be published and due effort will be made to conceal their identity, but that anonymity cannot be guaranteed.

\section{REFERENCES}

1. Mevorah B, Frenk E, Saurat JH, Siegenthaler G. Peeling skin syndrome: A clinical, ultrastructural and biochemical study. Br J Dermatol. 1987;116:117-25.

2. Verma K, Sharma RK, Gupta M, Thakur S. EDSF (McGrath syndrome) - A rare variant of epidermolysis bullosa simplex. Our Dermatol Online. 2019;10:281-3.

3. Gopal Anoop DS, Samayam A, Bijina KD. Ichthyoses: Case series. Our Dermatol Online. 2018;9:190-3.

4. Szczecinska W, Nesteruk D, Wertheim-Tysarowska K, Greenblatt DT, Baty D, Browne F, et al. Under-recognition of acral peeling skin syndrome: 59 new cases with 15 novel mutations. Br J Dermatol. 2014;171:1206-10.

Copyright by Sanae Sialiti, et al. This is an open access article distributed under the terms of the Creative Commons Attribution License, which permits unrestricted use, distribution, and reproduction in any medium, provided the original author and source are credited.

Source of Support: Nil, Conflict of Interest: None declared. 